\title{
Alteração nos atributos químicos de um Latossolo Amarelo pela calagem superficial em área sob cultivo de manga
}

\section{Alteration of chemical attributes of a yellow oxisol by surface liming in area under mango crop}

\author{
Fernando Silva Araújo ${ }^{1 *}$; Adeodato Ari Cavalcante Salviano²; \\ Aluizio Duarte Coelho Filho ${ }^{3}$
}

\begin{abstract}
Resumo
O presente estudo teve como objetivo avaliar o efeito de diferentes doses de calcário, aplicadas na superfície do solo sobre a acidez e os teores de $\mathrm{Ca}^{2+}$ e $\mathrm{Mg}^{2+}$ em diferentes profundidades, cultivado com manga. $\mathrm{O}$ experimento foi conduzido em pomar localizado município de Teresina, PI, em campo experimental da Escola Família Agrícola do Soinho. O delineamento experimental empregado foi o inteiramente casualizado com quatro tratamentos, doses de calcário de $0 ; 0,5 ; 1,0$ e 2,0 $\mathrm{Mg} \mathrm{ha}^{-1}$, e quatro repetições totalizando 16 parcelas de $80 \mathrm{~m}^{2}$ cada. As amostragens foram realizadas aos quatro, oito e dezesseis meses após a aplicação do calcário. Em cada parcela foram retiradas quatro amostras simples para formar uma amostra composta nas profundidades $(0,00-0,05 ; 0,05-0,10 ; 0,10-0,15$ e $0,15-$ $0,20 \mathrm{~m}$ ). Os resultados obtidos foram submetidos à análise de variância e de regressão, de acordo com os procedimentos do software SAS. O efeito da aplicação de calcário em superfície sobre o $\mathrm{pH}, \mathrm{Al}^{3+}, \mathrm{H}^{+}$ $+\mathrm{Al}^{3+}$ e teor de $\mathrm{Mg}^{2+}$ foi restrito à camada superficial do solo e, mostrou se intensificar com o tempo.

Palavras-chave: Acidez, $\mathrm{Ph}$, doses de calcário, Mangifera indica L
\end{abstract}

\begin{abstract}
This study was developed to evaluate the effect of different limestone rates on the soil acidity and $\mathrm{Ca}^{2+}$ and $\mathrm{Mg}^{2+}$ contents in different depths, when the limestone was applied in to surface of the soil, in an area already implanted with the mango crop. The experiment was accomplished in the experimental field of Soinho's Agricultural Family School, in Teresina, PI. The experimental design was completely randomized with four treatments based in the limestone levels: $0 ; 0.5 ; 1.0$ and $2.0 \mathrm{Mg} \mathrm{ha}^{-1}$, and four replications totalizing 16 plots, with $80 \mathrm{~m}^{2}$ each. The samplings were accomplished at four, eight and sixteen months after the application of the limestone. In each plot four simple samples were collected to form one bulked sample in the depths $(0.00-0.05 ; 0.05-0.10 ; 0.10-0.15$ and $0.15-0.20 \mathrm{~m})$. The results were submitted to the variance and regressions analysis, according to the procedure of the SAS software. The effect of the limestone application in the soil surface on $\mathrm{pH}, \mathrm{Al}^{3+}, \mathrm{H}^{+}, \mathrm{Al}^{3+}$ and $\mathrm{Mg}^{+2}$ contents were intensified in function of the time.
\end{abstract}

Key words: Acidity, $\mathrm{Ph}$, limestone levels, Mangifera indica $L$

1 M. Sc. Agronomia, Doutorando da Universidade Estadual de Campinas (UNICAMP). E-mail: fernandophb@oi.com.br

2 Dr. em Ciência do Solo, Prof. do Centro de Ciências Agrárias da (UFPI). E-mail: asalviano@uol.com.br

3 Eng. Agr. Aluizio Duarte Coelho Filho, Incra/Santarém.. E-mail: aluizio.duarte@sta.incra.gov.br

* Autor para correspondência 


\section{Introdução}

$\mathrm{Na}$ região da grande Teresina, PI tem-se intensificado a exploração da fruticultura irrigada, sendo a cultura da manga uma das mais trabalhadas. No entanto, pouca atenção tem sido dada ao aspecto do manejo do solo, principalmente no que se refere à correção de acidez e níveis de cálcio e magnésio.

Os solos das regiões tropicais e subtropicais, em sua grande maioria são, normalmente, ácidos e apresentam teores elevados de alumínio trocável (CIOTTA et al., 2004; COSTA; ROSOLEM, 2007). Nessas condições, a deficiência de $\mathrm{Ca}$ e a toxidez por Al são as principais limitações químicas para o crescimento radicular, cujas conseqüências se manifestam pelo estresse nutricional e hídrico das plantas (RITCHEY et al., 1980; ZAMBROSI; ALLEONI; CAIRES, 2007). Como alternativa para viabilização do cultivo racional desses solos faz-se o uso da calagem, que promove a elevação do $\mathrm{pH}$ e redução dos teores de alumínio trocável, além de aumentar os teores de cálcio e magnésio e a capacidade de troca de cátions do solo proporcionando um incremento de produção para as culturas (CAIRES et al., 2008).

O método convencional para a aplicação de calcário ao solo, por meio da incorporação mecânica do calcário ao solo, garante distribuição uniforme do corretivo nas camadas superficiais (PÁDUA; SILVA; MELO, 2006), no entanto, devido à baixa solubilidade do calcário, essa prática necessita de revolvimento do solo, para aumentar a área superficial de contato e a ação neutralizante do calcário, além disso, a calagem profunda pode vir a exigir máquinas potentes e equipamentos caros, o que torna a prática onerosa, razão pela qual não é de interesse em áreas com culturas já estabelecidas (CAIRES et al., 1998).

A aplicação superficial de corretivos pode contribuir para a correção eficiente da acidez do solo, principalmente em áreas já instaladas com culturas perenes (CHAVES; PAVAN; IGUE, 1984). Contudo, a aplicação superficial de calcário, sem posterior incorporação ao solo do mesmo pode vir a resultar numa correção inadequada da acidez nas camadas mais profundas de solo exploradas pelas raízes da cultura (PÁDUA; SILVA; MELO, 2006).

No Estado do Piauí são escassos os trabalhos que visam elucidar sobre o melhor método de aplicação de calcário em culturas perenes, bem como sobre a reação do calcário aplicado na superfície do solo. Essas informações são, particularmente, importantes para as áreas implantadas com fruteiras permanentes, no estado do Piauí, considerando que a textura predominante nos solos dessa região é de arenosa para média (JACOMINE et al., 1986).

O presente estudo teve como objetivo avaliar o efeito de diferentes doses de calcário sobre a

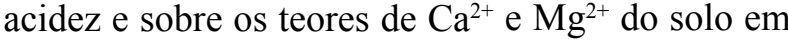
diferentes profundidades, quando este foi aplicado na superfície do solo, em uma área já implantada com a cultura da manga.

\section{Material e métodos}

O experimento foi conduzido em pomar de manga localizado entre os paralelos de $4^{\circ} 54^{\prime}$ e $5^{\circ} 00^{\prime} \mathrm{S}$ e meridianos de $42^{\circ} 40^{\prime}$ e $42^{\circ} 46^{\prime}$ a oeste de Greenwich, no município de Teresina - PI, em campo experimental da Escola Família Agrícola do Soinho. A área total usada no experimento foi de $1.280 \mathrm{~m}^{2}$ e o espaçamento utilizado no pomar de 10,0 x 8,0 m. O solo da área é classificado, de acordo com SiBCS, como um Latossolo Amarelo (EMPRESA BRASILEIRA DE PESQUISA AGROPECUÁRIA, 1999). O clima da região é do tipo Aw’ no sistema de Koppen, a precipitação média anual é de $1.448 \mathrm{~mm}$, a temperatura média anual de $27,3^{\circ} \mathrm{C}$ e a umidade relativa do ar média anual de $70 \%$. Na Tabela 1 é apresentada a caracterização química do solo, antes da instalação do experimento segundo metodologia de Embrapa (1997). 
Tabela 1. Caracterização química do Latossolo Amarelo, na camada de $0-0,20 \mathrm{~m}$, antes da instalação do experimento.

\begin{tabular}{lllllllll}
\hline $\mathbf{p H}$ & $\mathbf{C a}$ & $\mathbf{M g}$ & $\mathbf{K}$ & Al & H+Al & $\mathbf{C T C}$ & $\mathbf{S}$ & $\mathbf{V \%}$ \\
\hline $\mathrm{H}_{2} \mathrm{O}$ & $\mathrm{cmol}_{\mathrm{c}} \mathrm{dm}^{-3}$ & & & & & & & \\
\hline 5,7 & 2,27 & 1,48 & 0,20 & 0,07 & 2,85 & 6,79 & 3,94 & 58 \\
\hline
\end{tabular}

O delineamento experimental empregado foi o inteiramente casualizado com quatro tratamentos (doses de calcário de $0 ; 0,5 ; 1,0$ e 2,0 $\mathrm{Mg} \mathrm{ha}^{-1}$ ) e quatro repetições totalizando 16 parcelas. Cada parcela experimental possuía área de 80 $\mathrm{m}^{2}$. As doses de calcário com valores médios de $95 \%$ de poder relativo de neutralização total (PRNT) foram aplicadas manualmente, à lanço, na superfície do solo, sem incorporação, no dia 13 de janeiro de 2003.

As amostragens do solo, para efeito da avaliação da reação do calcário no solo, foram realizadas aos quatro, oito e dezesseis meses após a aplicação do calcário. Em cada parcela foram retiradas quatro amostras simples para formar uma amostra composta nas profundidades $(0,0-0,05 ; 0,05-0,10 ; 0,10-$ $0,15$ e $0,15-0,20 \mathrm{~m})$. Em cada amostra de solo foram determinados o pH em água (1:2,5 relação

(a)

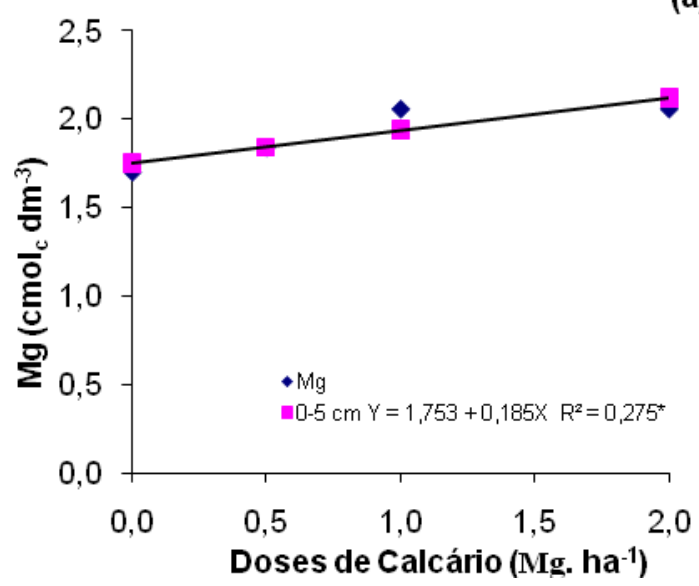

solo:água) e teores de $\mathrm{Ca}^{2+}, \mathrm{Mg}^{2+}, \mathrm{Al}^{3+} \mathrm{e} \mathrm{H}^{+}+\mathrm{Al}^{3+}$ (EMBRAPA, 1997).

Os resultados obtidos foram submetidos à análise de variância, e quando significativos foram aplicadas análise de regressões. Para realização das análises estatísticas utilizou-se o software SAS (Statiscal Analytical System).

\section{Resultados e discussão}

Os resultados obtidos demonstraram efeitos significativos, na camada de 0,0 - 0,05 $\mathrm{m}$ de profundidade para a maioria dos atributos estudados, excetuando-se para o Ca. Quando da avaliação após quatro meses da aplicação de calcário observou-se efeito significativo para os teores de $\mathrm{Mg}^{2+}(\rho<0,05)$ $\mathrm{e}^{+}+\mathrm{Al}^{3+}(\rho<0,01)$, havendo aumento nos teores de $\mathrm{Mg}^{2+}$ e decréscimo na acidez potencial com o aumento da dose de calcário aplicada (Figuras 1a e 1b).

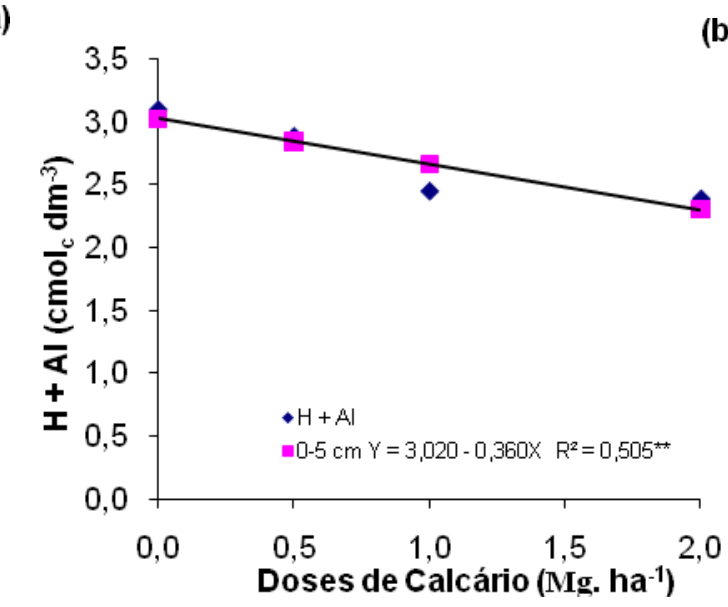

Figura 1. Efeito da aplicação de calcário na superfície do solo após quatro meses sobre os teores de $\mathrm{Mg} 2+(\mathrm{a})$ e acidez potencial (b), na profundidade de $0,0-0,05 \mathrm{~m}$. * Significativo $\rho<0,05$ e **Significativo $\rho<0,01$ 
Ausência do efeito de calcário sobre o $\mathrm{pH}$ decorre, provavelmente, do pouco tempo de reação do calcário, visto que o ânion acompanhante do cálcio $\left(\mathrm{CO}_{3}^{-2}\right)$ é uma base fraca, que se reflete em uma lenta liberação de $\mathrm{OH}^{-}$, ânion responsável pela neutralização dos íons $\mathrm{H}^{+}$na solução do solo. Outro aspecto a ser considerado é que a ação do calcário em profundidade, aplicado a partir da superfície do solo, é restrita, pois os ânions resultantes da sua dissolução, responsáveis pela neutralização da acidez, são consumidos nas reações com outros cátions ácidos $\left(\mathrm{Fe}^{2+}, \mathrm{Mn}^{2+}\right.$ e $\left.\mathrm{Al}^{3+}\right)$ na camada de deposição do calcário (MIYAZAWA; PAVAN;
SANTOS, 1996; ERNANI; RIBEIRO; BAYER, 2001; COSTA; ROSOLEM, 2007). Tal fato evidencia que a ação do produto em profundidade somente deve ocorrer após a neutralização dos cátions ácidos próximos a região de aplicação.

A avaliação após oito meses mostrou comportamento semelhante ao observado nos quatro meses iniciais, aumento de $\mathrm{pH}$ (Figura 2 a) com elevação das doses aplicadas, bem como, diminuição nos teores de alumínio trocável (Figura 2 b), o que também foi observado por Pires et al. (2003).
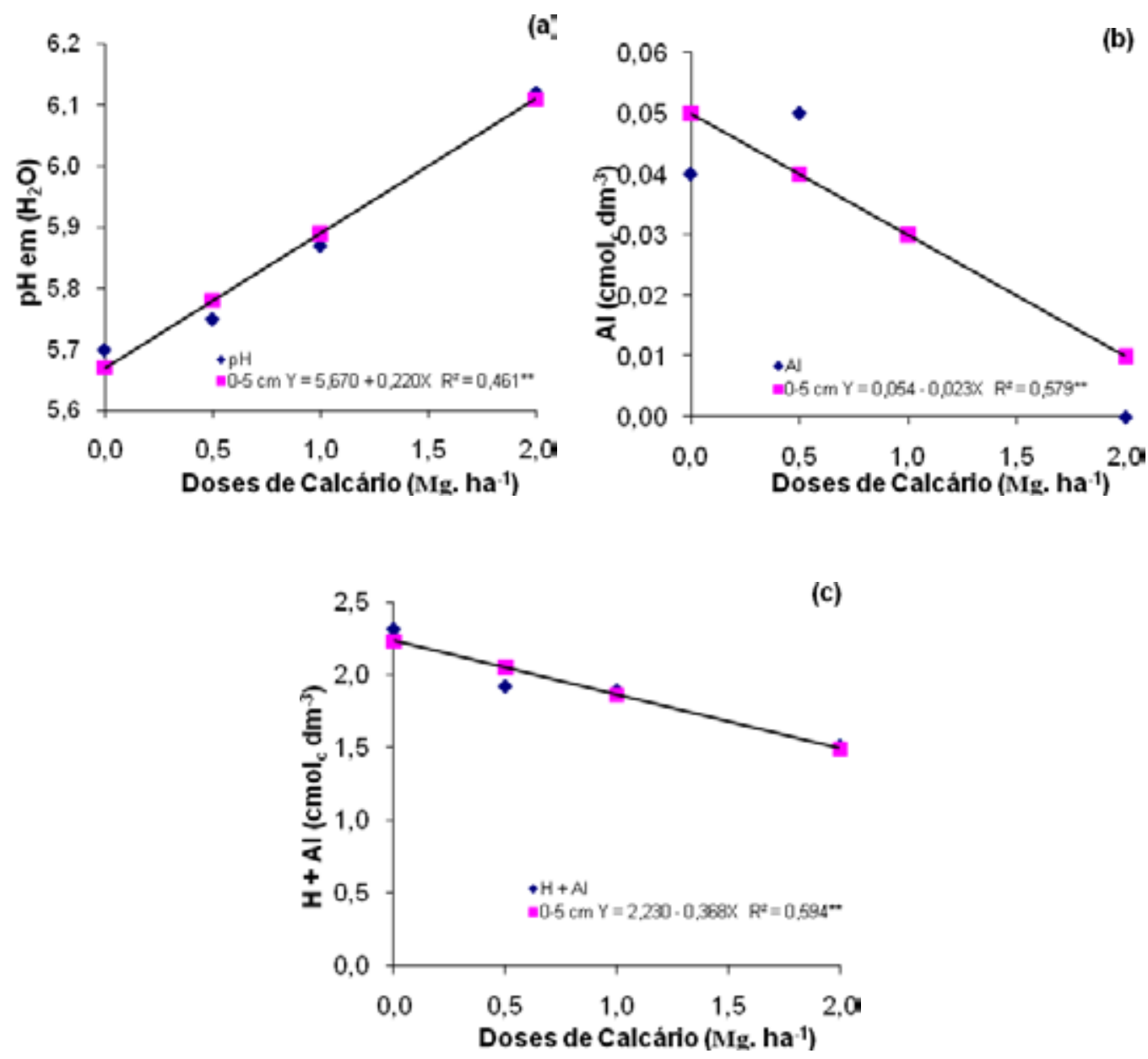

Figura 2. Efeito da aplicação de calcário na superfície do solo após oito meses sobre o pH (a), teores de Al3+ (b) e acidez potencial $(\mathrm{H}++\mathrm{Al} 3+)(\mathrm{c})$, na profundidade de $0,0-0,05 \mathrm{~m}$. ** Significativo $\rho<0,01$ 
$\mathrm{O}$ teor de $\mathrm{H}^{+}+\mathrm{Al}^{3+}$, após oito meses da aplicação do calcário, reduziu significativamente $(\rho<0,01)$ com o aumento das doses de calcário (Figura $2 \mathrm{c}$ ).

Mesmo após 16 meses da aplicação do calcário, não se verificou efeito em profundidade maior que $5 \mathrm{~cm}$. Isso mostra que a aplicação de calcário na superfície do solo estudado ficou restrita à camada superficial do solo, o que corrobora com observado por Caires et al. (2008). No caso do pH (Figura 3 a) já se verifica uma estabilização de seus valores com o aumento da dose, provavelmente devido a neutralização do $\mathrm{Al}^{3+}$, pois o efeito para esta variável não foi verificado, além do acentuado decréscimo nos valores de $\mathrm{H}^{+}+\mathrm{Al}^{3+}$ aos 16 meses (Figura $3 \mathrm{c}$ ).
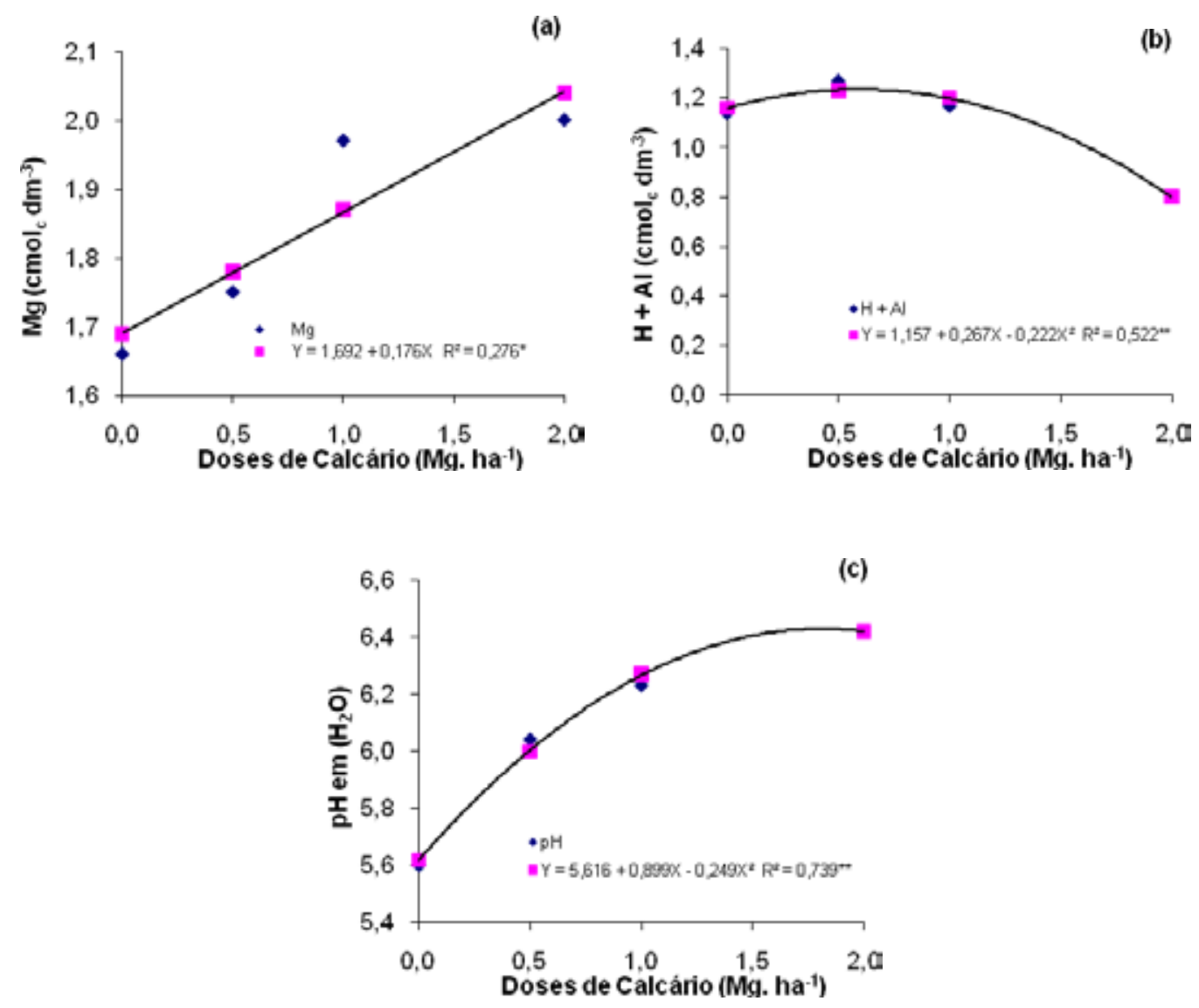

Figura 3. Efeito da aplicação de calcário na superfície do solo após 16 meses no $\mathrm{pH}$ (a), teores de $\mathrm{Mg} 2+$ (b) e $\mathrm{H}++$ $\mathrm{Al} 3+(\mathrm{c})$, na profundidade de $0,0-0,05 \mathrm{~cm}$. $*$ Significativo $\rho<0,05 . * *$ Significativo $\rho<0,01$

$\mathrm{O} \mathrm{Mg}^{2+}$ por sua vez manteve uma tendência de aumento com a elevação da dose aplicada. O efeito positivo da calagem no aumento do $\mathrm{pH}$, teores de $\mathrm{Mg}^{2+}$ e redução da acidez potencial $\left(\mathrm{H}^{+}+\mathrm{Al}^{3+}\right)$ foram verificados por Marcelo, Corá e Fernandes (2004). Caires et al. (2003), observaram que o calcário aplicado na superfície apresenta eficiência na correção da acidez de camadas superficiais do solo, pois sua ação na neutralização da acidez do subsolo é dificultada pelo aumento da retenção de cátions, em decorrência da geração de cargas elétricas variáveis negativas com a elevação do $\mathrm{pH}$ do solo. 
Fidalski e Tormena (2005), ao estudarem a dinâmica da calagem superficial em um Latossolo Vermelho-distrófico verificaram que após 37 meses da aplicação de calcário em superfície, é que foi evidenciada a máxima alteração dos atributos químicos do solo, em doses superiores ao dobro da necessidade de calcário, convencionalmente determinada, pelo método de saturação por bases. $\mathrm{Na}$ situação do presente estudo a correção da acidez do solo $\left(\mathrm{pH}\right.$ e $\left.\mathrm{H}^{+}+\mathrm{Al}^{+3}\right)$ ocorreu até a profundidade de $0,10-0,20 \mathrm{~m}$. Petrere e Anghinoni (2001) constataram que após 42 meses da aplicação superficial de calcário, a ação desse material em relação ao $\mathrm{pH}$, teve efeito até camada à $0,10-0,15 \mathrm{~m}$ de profundidade.

Os resultados obtidos no presente estudo mostraram que a eficiência da calagem na neutralização da acidez até os 16 meses da aplicação do produto só foi observada na camada de $0,0-0,05$ $\mathrm{m}$ de profundidade. Isto se deve, provavelmente, à baixa solubilidade dos corretivos agrícolas de acidez e à alta reatividade de seus ânions com os ácidos presentes na camada de solo em que o calcário é aplicado, o que contribui para que os valores de $\mathrm{pH}$ e Al alterem-se pouco em profundidade, quando da aplicação superficial de calcário. Enquanto, a baixa mobilidade de $\mathrm{Ca}$ e $\mathrm{Mg}$, por outro lado, deve-se à pequena permanência dos ânions adicionados pelo calcário na solução do solo. Sendo assim, a quase totalidade do $\mathrm{Ca}$ e do $\mathrm{Mg}$ adicionada vai para as cargas negativas criadas pelo aumento do $\mathrm{pH}$, e para as cargas anteriormente ocupadas pelo Al, e só uma pequena parte fica na solução do solo (ERNANI; RIBEIRO; BAYER, 2001).

Avaliações realizadas num período de tempo maior poderão mostrar, provavelmente, efeitos da aplicação do produto também em profundidade conforme relatado por Costa e Rosolem (2007) que após 36 meses observaram efeito da aplicação superficial de calcário até $60 \mathrm{~cm}$ de profundidade.

\section{Conclusões}

$\mathrm{O}$ efeito da aplicação de calcário em superfície sobre o $\mathrm{pH}, \mathrm{Al}^{3+}, \mathrm{H}^{+}+\mathrm{Al}^{3+}$ e teor de $\mathrm{Mg}^{2+}$ foi restrito à camada superficial do solo e, mostrou se intensificar com o tempo.

\section{Referências}

CAIRES, E. F.; KUSMAN, M. T.; BARTH, G.; GARBUIO, F. J.; PADILHA, J. M. Alterações de características químicas do solo e resposta da soja ao calcário e gesso aplicados na superfície em sistema de cultivo sem preparo do solo. Revista Brasileira de Ciência do Solo, Campinas, v. 22, n. 1, p. 27-34, 1998.

CAIRES, E. F.; BLUM, J.; BARTH, G.; GARBUIO, F. J.; KUSMAN, M. T. Alterações químicas do solo e resposta da soja ao calcário e gesso aplicados na implantação do sistema de plantio direto. Revista Brasileira de Ciência do Solo, Campinas, v. 27, n. 2, p. 275-286, 2003.

CAIRES, E. F.; GARBUIO, F. J.; CHURKA, S.; BARTH, G.; CORREAA, J. C. L. Effects of soil acidity amelioration by surface liming on no-till corn, soybean, and wheat root growth and yield. European Journal of Agronomy, Amsterdam, v. 28, n. 1, p. 57-64, 2008.

CHAVES, J. C. D.; PAVAN, M. A.; IGUE, K. Resposta do cafeeiro à calagem. Pesquisa Agropecuária Brasileira, Rio de Janeiro, v. 19, n. 5, p. 573-582, 1984.

CIOTTA, M. N.; BAYER, C.; ERNANI, P. R.; FONTOURA, S.M.V.;WOBETO, C.;ALBUQUERQUE, J. A. Manejo da calagem e os componentes da acidez de Latossolo bruno em plantio direto. Revista Brasileira de Ciência do Solo, Campinas, v. 28, n. 2, p. 317-326, 2004.

COSTA, A.; ROSOLEM, C. A. Liming in the transition to no-till under a wheat-soybean rotation. Soil \& Tillage Research, Amsterdam, v. 97, n. 2, p. 207-217, 2007.

EMPRESA BRASILEIRA DE PESQUISA AGROPECUÁRIA - EMBRAPA. Centro Nacional de Pesquisa de Solos. Manual de métodos de análise de solos. 2. ed. Rio de Janeiro: EMBRAPA, 1997. 212 p. (EMBRAPA-CNPS. Documentos, n. 1).

EMPRESA BRASILEIRA DE PESQUISA AGROPECUÁRIA - EMBRAPA. Centro Nacional de Pesquisa de Solos. Sistema brasileiro de classificação de solos. Rio de Janeiro: EMBRAPA, 1999. 412 p. 
ERNANI, P. R.; RIBEIRO, M. S.; BAYER, C. Modificações químicas em solos ácidos ocasionadas pelo método de aplicação de corretivos da acidez e de gesso agrícola. Scientia Agricola, Piracicaba, v. 58, n. 4, p. $825-$ 831, 2001.

FIDALSKI, J.; TORMENA, C. A. Dinâmica da calagem superficial em um Latossolo Vermelho distrófico. Revista Brasileira de Ciência do Solo, Campinas, v. 29, n. 2, p. 235-247, 2005.

JACOMINE, P. K. T.; CAVALCANTI, A. C.; PESSOA, S. C. P.; BURGOS, N.; MELO FILHO, H. F. R.; LOPES, O. F.; MEDEIROS, L. A. R. Levantamento exploratórioreconhecimento de solos do Estado do Piaui. Rio de Janeiro: EMBRAPA-SNLCS, SUDENE-DRN, 1986. 782 p.

MARCELO, A. V.; CORÁ, J. E.; FERNANDES, C. Aplicação superficial de calcário e absorção foliar da soja em sistema de plantio direto. In: REUNIÃO BRASILEIRA DE MANEJO E CONSERVAÇÃO DO SOLO E DA ÁGUA, 15., 2004, Santa Maria. Anais... Santa Maria, 2004.

MIYAZAWA, M.; PAVAN, M. A.; SANTOS, J. F. C. Effects of addition of crops residues on the leaching of $\mathrm{Ca}$ and $\mathrm{Mg}$ in Oxisols. In: INTERNATIONAL SYMPOSIUM ON PLANT-SOIL INTERACTIONS AT LOW pH, 4., 1996, Belo Horizonte. Anais... Belo
Horizonte: Sociedade Brasileira de Ciência do Solo, EMBRAPA-CPAC, 1996. p. 8.

PÁDUA, T. R. P.; SILVA, C. A.; MELO, L. C. A. M. Calagem em Latossolo sob influência de coberturas vegetais: neutralização da acidez. Revista Brasileira de Ciência do Solo, Campinas, v. 30, n. 5, p. 869-878, 2006.

PETRERE, C.; ANGHINONI, I. Alteração de atributos químicos no perfil do solo pela calagem superficial em campo nativo. Revista Brasileira de Ciência do Solo, Campinas, v. 25, n. 4, p. 885-895, 2001.

PIRES, F. R.; SOUZA, C. M.; QUEIROZ, D. M.; MIRANDA, G. V.; GALVÃO, J. C. C. Alteração de atributos químicos do solo e estado nutricional e características agronômicas de plantas de milho, considerando as modalidades de calagem em plantio direto. Revista Brasileira de Ciência do Solo, Campinas, v. 27, n. 1, p. 121-131, 2003.

RITCHEY, K. D.; SOUZA, D. M. G.; LOBATO, E.; CORREA, O. Calcium leaching to increase rooting depth in a Brazilian Savannah Oxisol. Agronomy Journal, Madison, v. 72, n. 1, p. 40-44, 1980.

ZAMBROSI, F. C. B.; ALLEONI, L. R. F.; CAIRES, E. F. Gypsum application and ionic speciation of the solution from an Oxisol under no-till system. Ciência Rural, Santa Maria, v. 37, n. 1, p. 110-117, 2007. 
